\title{
Perfil de los cuidadores informales de personas con enfermedades crónicas y calidad de vida, Villavicencio, Meta, 2011
}

\author{
Chronic patients' caregivers profile and quality of life, \\ Villavicencio, Meta, 2011
}

\section{Perfil dos cuidadores informais de pessoas condoencas crônica e qualidade de vida, Villavicencio, Meta, 2011}

María L. Pinzón - Rocha ${ }^{*}$, Luz H. Aponte - Garzón ${ }^{2 *}$, Clara R. Galvis - López ${ }^{3}$

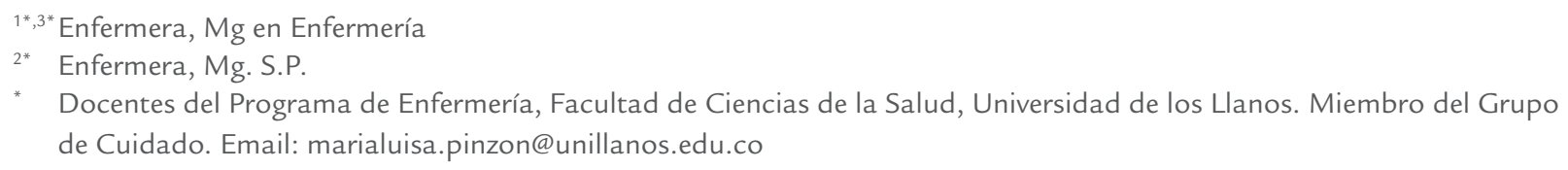

Recibido: febrero 14 de $2012 \quad$ Aceptado: junio 13 de 2012

\begin{abstract}
Resumen
Con el objeto de describir el perfil de los cuidadores informales de personas en cronicidad y la relación con la calidad de vida, se diseñó y desarrolló un estudio descriptivo de tipo cuantitativo, observacional, transversal. Los participantes ( $\mathrm{n}=180$ ) fueron seleccionados por método no probabilístico de tipo intencional, del conjunto de cuidadores informales de personas con enfermedad crónica inscritas en el programa de Promoción y Prevención del Adulto Crónico de la Empresa Social del Estado del municipio de Villavicencio, Meta. La recolección de información se hizo con un instrumento diseñado para tal fin que valora el perfil social, educativo, de salud y de desempeño del rol de cuidador. La medición de calidad de vida se hizo a partir de la aplicación del instrumento de calidad de vida de B. Ferrell. Los resultados muestran que la mayoría de los cuidadores son mujeres (79\%), con edad promedio de 46,02 años (DS= $=15,72$ ), esposas o hijas de los enfermos crónicos, con niveles educativos bajos (entre primaria a bachillerato incompleto 64\%), económicamente dependientes, quienes informan $(86 \%)$ que no han recibido capacitación para ser cuidadores informales. Los cuidadores son cuidadores únicos quienes ofrecen cuidado permanente. Este aspecto mostró relación protectora significativa con una buena calidad de vida del cuidador (OR: 0,45; LC:0,20-0,97;X2=4,87; valor p:<0,05). El cuidador informal muestra en general respuesta adaptativa positiva a su rol pero demanda gran inversión de tiempo y esfuerzo por lo que se recomienda un acompañamiento para mantener la calidad de vida del cuidador y la mejor condición del enfermo crónico en su entorno domiciliario.
\end{abstract}

Palabras clave: cuidadores, atención domiciliaria en salud, enfermedad crónica.

\begin{abstract}
A descriptive cross-sectional study was carried out in order to describe informal caregivers' profile and the relation with their quality of life. The participants $(n=180)$ were selected by non-probabilistic method, from chronic patients' informal caregivers registered in the Promotion and Prevention Program for Chronic Adults at the municipality of Villavicencio, Meta. Information was collected using an instrument designed for the study to assess social, educational, health and caregiver's performance profiles. Quality of life measurements were done applying the Quality of Life Instrument by B. Ferrell. Results
\end{abstract}


showed that most of the caregivers were women (79\%), with mean age of 46,02 year-old (DS= $\pm 15,72)$, they were related to the patient, being mainly spouses or daughters, had low educational levels (between primary school to incomplete high school education, 64\%), caregivers are economically dependents $(86 \%)$ and they have not received any training to be informal caregivers. Informal caregivers are the only and permanent domiciliary care source. This variable showed to be a significant protector factor for quality of life among caregivers (OR: 0,45; LC:0,20-0,97;X2=4,87; valor p: <0,05). Informal caregivers in general, reported positive response to their care role, but demand increased time and effort. For this, it is recommended to offer the needed support in order to maintain caregivers' quality of life and the best possible condition of chronic patient at his/her home environment.

Key Words: Caregiver, home care service, chronic disease.

\begin{abstract}
Resumo
Como o objetivo de descrever o perfil dos cuidadores informais de pessoas com cronicidade e a relação com a qualidade de vida foi realizado um estudo descritivo, quantitativo, observacional, transversal. Os participantes ( $n=180)$ foram selecionados por método no probabilístico de tipo intencional do conjunto de cuidadores informais de pessoas com doença crônica inscritas no programa de Promoção e Prevenção do adulto crônico da Empresa Social do estado do município de Villavicencio, Meta. A coleta de informação foi realizada com instrumento projetado e que valora o perfil social, educativo, de saúde e desempenho do papel de cuidador. A medição da qualidade de vida foi realizada pela aplicação do instrumento de qualidade de vida de B. Ferrell. Os resultados amostram que a maioria dos cuidadores são mulheres (79\%), e idade media de 46,02 15,72 anos, esposas o filhas dos doentes crônicos, com nível educativo baixo (64\% com ensino básico até médio incompleto) e que não tem recebido treinamento para serem cuidadores informais. Os cuidadores são únicos e oferecem atenção permanente. Este aspecto apresentou relação protetora significativa com boa qualidade de vida do cuidador (OR:0,45; LC:0,20-0,97;X2=4,87; valor $\mathrm{p}:<0,05)$. O cuidador informal apresenta em geral resposta adaptativa positiva ao seu papel, porém, requer dedicação em tempo e esforço, por este motivo recomenda-se acompanhamento para manter a qualidade de vida do cuidador e a melhor condição do doente crônico no seu entorno domiciliário.
\end{abstract}

Palavras Chave: Cuidadores, Atenção domiciliária em saúde, doença crônica.

\section{Introducción}

A pesar de los avances tecnológicos, científicos y las estrategias desarrolladas por el sector salud en relación a la promoción y el cuidado de la salud, el cuidado informal sigue siendo la forma más predominante y fundamental para el cuidado de las enfermedades crónicas o de larga duración, que ocupan cada vez un lugar más preponderante en el perfil epidemiológico de los países desarrollados y en vías de desarrollo (Carretero 2006, Romero et.al. 2011). Esta responsabilidad que requiere más de cuidado en donde generalmente hay una persona integrante de la familia, llamado "cuidador principal", que asume la responsabilidad del cuidado de la persona enferma, en forma permanente y en la mayoría de los casos, sin ninguna preparación previa para asumir y desempeñar este rol (Crespo 2007).

Cuidar significa, dedicación, compromiso, conocimiento, experiencia, afecto, responsabilidad, vigilancia, preocupación y ayuda a otro a lograr su realización personal (González et.al). En este sentido se hace necesario analizar algunas características del cuidador informal que son determinantes en el proceso de cuidar para brindar un cuidado eficaz y oportuno, centrado en las necesidades de los sujetos de cuidado, con el fin de promover la salud, prevenir la aparición de complicaciones, evitando el deterioro de la salud y de la calidad de vida.

Caracterizar al cuidador informal y analizar aspectos fundamentales que contribuyan a la calidad del cuidado domiciliario, debe ser una prioridad para los profesionales de la salud y principalmente para Enfermería, teniendo en cuenta que el cuidador informal es el puente entre el paciente y el servicio de salud y el responsable directo de realizar intervenciones de cuidado, dirigidas a realizar un cuidado eficaz a partir de la promoción de la salud y prevención de complicaciones propias de la enfermedad. De igual forma la orientación del cuidado y el desarrollo de potencialidades en el cuidador informal, favorece la comprensión de la enfermedad y el desarrollo de habilidades para el cuidado de sí mismo y de otros, con el fin de fortalecer desempeño del rol y favorecer el autocuidado, el bienestar y la calidad de vida de los involucrados en situaciones de cronicidad (Ocampo 2007). De acuerdo a lo anterior, se propuso en este trabajo, examinar las características socio-demográficas y de salud del cuidador, y las condiciones en las que el cuidador ofrece el cuidado informal al enfermo crónico en la ciudad de Villavicencio, Meta, aspectos determinantes para el desempeño eficaz del rol. 


\section{Materiales y métodos}

Se realizó un trabajo descriptivo de tipo cuantitativo, observacional, transversal, con cuidadores informales, pertenecientes a los programas de Promoción y Prevención del Adulto Crónico de seis Centros de Salud de la ESE municipal de Villavicencio, Meta (Esperanza, Porfía, Morichal, Recreo, Popular y Porvenir,).

Los cuidadores de personas con enfermedad crónica que residen en el municipio de Villavicencio conformaron el universo y la muestra $(n=180)$ se compuso de cuidadores informales de personas con enfermedad crónica, y que tuvieran los criterios de inclusión nombrados a continuación: Ser mayor de edad ( $\geq 18$ años), ser cuidador informal de una persona en situación de enfermedad crónica inscrito en el Programa de Promoción y Prevención de una institución de salud vinculada a la Empresa Social del Estado (ESE) del municipio de Villavicencio, Meta y expresar por escrito su deseo de participar voluntariamente en el estudio, firmando el respectivo consentimiento informado según lo determina la Resolución 08430 de 1993 del Ministerio de Salud (MinSalud, 1993). Según esta resolución, la presente investigación se clasifica como investigación sin riesgo ya que la actividad investigativa está limitada a la aplicación anónima de un cuestionario sin realizar intervenciones ni modificaciones intencionadas de variables bio-psicosociales.

La selección de los participantes se hizo por método no probabilístico, de tipo intencional, siendo la unidad de muestreo cada cuidador informal participante en el estudio y la unidad de análisis la misma unidad de muestreo.

La recolección de la información se realizó a través de un instrumento diseñado para tal fin conformado por 31 preguntas agrupadas en cuatro categorías, cada una de las categorías contiene preguntas sobre un aspecto o perfil específico del cuidador. Los perfiles definidos en el instrumento fueron: 1) Perfil Social que incluye diez preguntas sobre variables demográficas y nivel socio-económico; 2) Perfil Educativo que incluye dos preguntas sobre nivel de escolaridad formal y sobre la capacitación recibida para el cuidado del enfermo crónico; 3) Perfil de Salud que contiene 8 preguntas e indaga sobre afiliación al sistema de salud del cuidador y percepción de su propia salud; y 4) Rol del Cuidador que incluye once preguntas sobre la edad y género del enfermo crónico que cuida y sobre aspectos relacionados con el cuidado dado, tales como tiempo de cuidado, parentesco con el paciente, participación de otros en el cuidado al enfermo, tipo de cuidados realizados y ayuda recibida para el cuidado.
El instrumento fue sometido a prueba piloto para asegurar la viabilidad de la medición estandarizando el tiempo de aplicación (15 minutos), la sencillez y claridad de las preguntas y la facilidad para la codificación y la interpretación de las respuestas. De igual manera, expertos en el tema de la investigación examinaron las preguntas y sus categorías para asegurar la validez lógica y de contenido. Los ajustes necesarios se realizaron dentro de la prueba piloto, procediendo a la recolección de la información.

Este instrumento fue aplicado a través de entrevista semi-estructurada, previa capacitación de Estudiantes Participantes en Investigación (EPI) del programa de Enfermería de la Universidad de los Llanos, por medio de talleres prácticos y previo consentimiento informado de los cuidadores informales participantes.

La medición de calidad de vida se hizo por medio del instrumento de Calidad de Vida de B. Ferrell. El instrumento está organizado en cuatro dimensiones, ellas son: física, psicológica, espiritual y social, con un total de 37 ítems que se responden usando escala de Likert con puntajes de 1 a 4 , siendo los puntajes resultantes posibles de 37 a 148 puntos. Este instrumento tiene un grado de validez y confiabilidad de 0.89 , y una consistencia de alfa $=0.69$ en los cuatro dominios. Un nivel de disminución de la calidad de vida se estableció en puntajes menores o iguales a 110 y calidad de vida sin disminución con puntajes iguales o mayores a 111. Los investigadores cuentan con la autorización del autor del instrumento para su uso en la investigación.

Para el análisis estadístico se utilizó el software Excel 2007 para Windows y el análisis de riesgo de realizó usando Epi Info 7. Las variables cuantitativas se describieron mediante medidas de tendencia central y dispersión. Las variables cualitativas se describieron con proporciones. Las asociaciones entre variables se exploraron usando estimación de riesgo (OR) con pruebas de Chi cuadrado a un nivel de significancia de 0,05.

\section{Resultados}

\section{Perfil social}

Los datos valorados dentro del perfil social (Tabla 1) señalan que la mayoría $(79 \%)$ de los cuidadores informales de la muestra pertenecen al género femenino, el $61 \%$ de los cuidadores participantes son adultos mayores de 40 años, son residentes de zona urbana en vivienda propia correspondiente a estrato socioeconómico de niveles 1 o 2. La distribución por edad muestra un promedio de 46,02 años (DS= $\pm 15,72$ ), un rango mínimo de 18 años y máximo de 84 años. En los cui- 
dadores de la muestra predomina (64\%) el estado civil de casados o unión libre.

Cerca de la mitad de los cuidadores participantes reportan estar dedicados al cuidado del hogar, de otro lado, el $66 \%$ de todos los cuidadores reportan tener ingresos propios, aún cuando las cantidades reportadas presentan amplia variación (valor min. \$50.000; valor máx. \$1'200.000). La mayoría de los cuidadores de la muestra reportan tener facilidad para relacionarse con otras personas en su entorno social, especialmente con miembros de la familia.

\section{Perfil educativo}

La Tabla 2, muestra que la escolaridad de los cuidadores informales está principalmente entre primaria incompleta y bachillerato incompleto siendo equivalente al $64 \%$ del total de cuidadores, y solo el $12 \%$ del total cuenta con formación de nivel técnico o profesional. En cuanto a la formación específica para el desempeño del rol, la mayoría manifestó no haber recibido ningún tipo de capacitación para desempeñar su rol de cuidador informal, el grupo que ha recibido alguna capacitación ha sido por parte de miembros del equipo de salud, en temas relacionados con alimentación, administración de medicamentos, cuidado en casa, manejo de la hipertensión arterial y diabetes, cambios de posición y ejercicios.

\section{Perfil de salud}

La Tabla 3, muestra que la mayoría de los cuidadores se encuentran afiliados al Sistema General de Seguridad Social en Salud (SGSSS), sin embargo, aún cuando es una pequeña proporción, se encontraron cuidadores informales que no cuentan con afiliación al SGSSS, es decir no cuentan con cobertura de servicios de salud. La mayoría de los cuidadores considera que su salud es buena, argumentando que pueden trabajar, que no tienen evidencia de enfermedad, pueden rea-

Tabla1. Perfil socioeconómico de los cuidadores informales, Villavicencio, 2011

\begin{tabular}{|c|c|c|c|}
\hline Variable & Categorías & Frecuencia & $\%$ \\
\hline \multirow{2}{*}{ Género } & Femenino & 142 & 79 \\
\hline & Masculino & 38 & 21 \\
\hline \multirow{3}{*}{ Edad (años) } & $18-40$ & 70 & 39 \\
\hline & 41- 60 & 75 & 42 \\
\hline & 61 en adelante & 35 & 19 \\
\hline \multirow[b]{2}{*}{ Ubicación Geográfica } & Urbana & 179 & 99 \\
\hline & Rural & 1 & 1 \\
\hline \multirow{2}{*}{ Tipo de Vivienda } & Propia & 128 & 71 \\
\hline & Arriendo & 52 & 29 \\
\hline \multirow{3}{*}{ Estrato Socioeconómico } & 1 & 45 & 25 \\
\hline & 2 & 84 & 47 \\
\hline & 3 & 51 & 28 \\
\hline \multirow{5}{*}{ Estado Civil } & Soltero & 38 & 21 \\
\hline & Casado & 68 & 38 \\
\hline & Separado & 17 & 9 \\
\hline & Viudo & 10 & 6 \\
\hline & U.Libre & 47 & 26 \\
\hline \multirow{5}{*}{ Ocupación } & Hogar & 87 & 48 \\
\hline & Empleado & 24 & 13 \\
\hline & T.Independiente & 63 & 35 \\
\hline & Estudiante & 5 & 3 \\
\hline & Otro & 1 & 1 \\
\hline \multirow{2}{*}{ Relación con otros } & $\mathrm{Si}$ & 168 & 93 \\
\hline & No & 12 & 7 \\
\hline
\end{tabular}


Tabla 2. Perfil educativo de los cuidadores informales, Villavicencio, 2011

\begin{tabular}{l|l|c|r}
\hline \multicolumn{1}{c|}{ Variable } & Rangos e indicadores & Frecuencia & $\%$ \\
\hline & Ninguna & 3 & 2 \\
& Primaria incompleta & 39 & 22 \\
& Primaria completa & 38 & 21 \\
& Bachillerato incompleto & 38 & 21 \\
& Bachillerato completo & 40 & 22 \\
& Formación técnica & 11 & 6 \\
& Universitaria incompleta & 7 & 4 \\
Capacitación específica & Universitaria completa & 4 & 2 \\
para el rol de cuidador & Si & 26 & 14 \\
\hline
\end{tabular}

lizar las actividades diarias y no han tenido necesidad de consultar al médico.

La gran mayoría del grupo de cuidadores participantes refieren que cuidan de su salud a través de visitas médicas, consumo de una adecuada alimentación, desarrollo de actividad física y buenos hábitos de higiene, aún cuando un poco más de la mitad de los participantes refiera tener síntomas o enfermedades que alteran su bienestar, siendo los más frecuentes dolor en cabeza y piernas, cansancio físico y estrés. La mayoría reconoce que en su cotidianidad, dedican entre 1 y 3 horas para el descanso, diferentes al sueño nocturno, y que ese tiempo lo dedican a actividades como ver televisión, dormir siesta, leer y dialogar con amigos.

\section{Desempeño del rol}

La Tabla 4 presenta la información relacionada con las condiciones en las que el cuidador ofrece el cuidado a la persona con enfermedad crónica. Los resultados muestran que la mayoría de personas son cuidadores únicos, es decir que no hay participación de otras personas en la atención del enfermo, por lo que hay una dedicación permanente en las acciones de cuidado.

Los cuidadores son en su mayoría familiares de los enfermos $(82 \%)$, siendo los vínculos más frecuentes ser esposas, hijas o nietas de los pacientes crónicos. Los enfermos tienen un promedio de edad de 66,8 años (DS $\pm 12,8$ años) con valores mínimo y máximo de 33 a 99 años respectivamente. A criterio de los cuidadores, la mayoría de personas en situación de cronicidad son independientes para realizar las actividades cotidianas, consideran que el desarrollo de la enfermedad se encuentra en etapas entre leve y moderado ( $42 \%$ y $48 \%$ ) respectivamente, solo el $9 \%$ se halla en un estado avanzado de deterioro el cual es progresivo, aspecto que genera mayor dependencia y cuidado.
Frente a la pregunta sobre los aspectos que facilitan el desempeño del rol de cuidador refieren que la existencia de un vínculo familiar, el afecto, el conocimiento personal, la convivencia, la disponibilidad de tiempo, el apoyo ocasional que reciben de otros miembros de la familia y el compartir, la misma enfermedad facilita el desarrollo de las acciones como cuidador informal.

El $18 \%$ que no se les facilita realizar este rol, manifestaron inconvenientes debido a que tienen su propia familia, viven en otra casa, trabajan, tienen múltiples actividades, no cuentan con recursos económicos suficientes, se sienten enfermos y consideran que las acciones de cuidado les demanda demasiado tiempo.

En cuanto a las actividades más comunes que realiza el cuidador, la mayoría refiere realizar el acompañamiento a citas médicas y compras, la administración de medicamentos, preparación de comidas, curaciones y ayuda en la realización de las actividades de la vida diaria, especialmente las relacionadas con las funciones de higiene personal y alimentación, entre otras.

La relación entre algunas condiciones importantes en las que se da el cuidado informal y la calidad de vida de quien cuida es presentada en la Tabla 5. En esta tabla se muestra de manera relevante el papel protector que tiene el cuidado permanente sobre la calidad de vida del cuidador informal, alcanzando un nivel estadísticamente significativo. Las otras asociaciones estudiadas tales como el grado de avance de la enfermedad y la capacidad de realización de actividades de la vida diaria (AVD) por parte del enfermo muestran asociación positiva de riesgo sobre la calidad de vida del cuidador, sin embargo los límites de confianza de la medida de riesgo incluyen la unidad y no alcanzan niveles de significancia estadística. Otras variables como cuidar de un enfermo mayor de 65 años y ser el 
Tabla 3. Perfil de salud de los cuidadores informales, Villavicencio, 2011

\begin{tabular}{l|l|r|r}
\hline \multicolumn{1}{c|}{ Variable } & \multicolumn{1}{c|}{ Categorías } & Frecuencia & \% \\
\hline \multirow{3}{*}{ Afiliación al SGSSS } & Si & 169 & 94 \\
& No & 11 & 6 \\
Percepción de la Salud & Buena & 112 & 62 \\
& Regular & 60 & 33 \\
& Mala & 8 & 4 \\
Cuidado de la Salud & Si & 159 & 88 \\
& No & 21 & 12 \\
Forma de cuidar la salud & Visita al médico & 118 & 27 \\
& Consumo adecuada alimentación & 116 & 27 \\
& Realiza ejercicio & 69 & 16 \\
Enfermedades o síntomas que alteran el bienestar & Tener buena higiene & 119 & 28 \\
& Otro & 9 & 2 \\
Dedicación de tiempo para el descanso & Si & 92 & 51 \\
& No & 88 & 49 \\
\hline
\end{tabular}

Tabla 4. Desempeño del rol del cuidador informal, Villavicencio, 2011

\begin{tabular}{|c|c|c|c|}
\hline Variable & Categorías & Frecuencia & $\%$ \\
\hline \multirow{9}{*}{ Parentesco con los sujetos de cuidado } & Esposo (a) & 46 & 26 \\
\hline & Hijos & 86 & 48 \\
\hline & Madre & 7 & 4 \\
\hline & Padre & 1 & 1 \\
\hline & Nieta & 14 & 8 \\
\hline & Sobrina & 1 & 1 \\
\hline & Amiga & 5 & 3 \\
\hline & Vecina & 2 & 1 \\
\hline & Otro & 18 & 10 \\
\hline \multirow{4}{*}{ Frecuencia del cuidado } & Días & 34 & 19 \\
\hline & Semanas & 7 & 4 \\
\hline & Meses & 2 & 1 \\
\hline & Permanente & 137 & 76 \\
\hline \multirow{2}{*}{ Único Cuidador } & $\mathrm{Si}$ & 109 & 61 \\
\hline & No & 71 & 39 \\
\hline \multirow{2}{*}{ Dependencia del sujeto de cuidado } & Independiente & 129 & 72 \\
\hline & Dependiente & 51 & 28 \\
\hline \multirow{2}{*}{ Se facilita la labor de cuidador } & $\mathrm{Si}$ & 147 & 82 \\
\hline & No & 33 & 18 \\
\hline
\end{tabular}


único que desempeña el rol de cuidador no muestran asociación positiva significativa.

\section{Discusión}

\section{Perfil social}

Las características sociales de los cuidadores informales de la ciudad de Villavicencio, coinciden con las descritas en otros estudios. (Espín 2008, Carretero et.al. 2006, Aravena et.al. 2010). El principal aspecto que se destaca es que la mayoría de cuidadores son mujeres, aspecto que reafirma el histórico papel asignado a este género frente al cuidado de la salud y de la vida del grupo familiar (Prieto 2007), otorgado fundamentalmente por la división de roles entre el hombre y la mujer, las expresiones de afecto, la identificación de valores, el amor filial y el deber moral; así mismo por su capacidad y dedicación de ser proveedora principal de los cuidados del hogar y responsable directa de la salud física y mental de cada uno de los miembros del grupo familiar; estos aspectos caracterizan el rol que debe asumir la mujer al interior de la familia.

Particularmente en el caso de ser proveedora de cuidados para el mantenimiento de la salud, la mayoría de estudios investigativos plantean que es desempeño de este rol se da en condiciones de inequidad, invisibilidad y exclusión (Vaquiro 2010), sin embargo algunos trabajos han demostrado que el cuidado brindado por mujeres es más significativo en cuanto a intensidad y frecuencia que el realizado por hombres (García 2004, Larrañaga 2008). Las características que rodean el cuidado brindado por mujeres se considera un escena- rio importante para fortalecer la calidad del cuidado a partir del reconocimiento de los seres humanos, las buenas relaciones familiares, la armonía, la dedicación, la empatía, el conocimiento de las personas, el valor, la paciencia y la ayuda en la satisfacción de necesidades, requisitos fundamentales para brindar un cuidado integral y humanizado (Bautista 2008).

Otro aspecto importante para el fortalecimiento del rol, son las edades de la mayoría de los cuidadores, las cuales se encuentran en la plenitud del desarrollo físico e intelectual para comprender las situaciones de salud, desarrollar el aprendizaje y mejorar la habilidad del cuidado. La formación y el conocimiento sobre conductas saludables y un manejo adecuado del estilo de vida en edades tempranas, contribuyen a minimizar los factores de riesgo para la salud del grupo familiar en general; así mismo se convierte en una tarea prioritaria para el personal de salud, teniendo en cuenta las responsabilidades que tiene el cuidador con el autocuidado y el desempeño efectivo del rol, evitando factores de riesgo que adicionen a futuro, graves repercusiones en la salud de sí mismo, del núcleo familiar y la sociedad en general (Guías Colombianas para el Diagnóstico y Tratamiento de la Hipertensión Arterial 2007, Qirantes et.al. 2009).

En cuanto a los adultos mayores que cuidan a personas de su misma edad y condición, se pueden ver enfrentados a diversos riesgos y limitaciones en el cuidado de sí mismo y de otros, debido a las incapacidades físicas y mentales propias de la edad y la demanda de cuidado por parte de la persona a quien cuidan (Gallardo et. al., 2004). Los cuidadores informales son

Tabla 5. Condiciones del rol del cuidador informal y su relación con la calidad de vida, Villavicencio, 2011

\begin{tabular}{|c|c|c|c|c|c|c|}
\hline \multirow{2}{*}{\multicolumn{2}{|c|}{ Condiciones del rol }} & \multicolumn{2}{|c|}{ Calidad de vida del cuidador } & \multirow{2}{*}{ OR (IC) } & \multirow{2}{*}{$X^{2}$} & \multirow{2}{*}{ Valor $p$} \\
\hline & & Disminuida & No disminuida & & & \\
\hline Edad del enfermo & $\begin{array}{l}>65 \text { años } \\
<=65 \text { años }\end{array}$ & $\begin{array}{l}52 \\
43\end{array}$ & $\begin{array}{l}50 \\
35\end{array}$ & $0,85(0,45-1,60)$ & 0,31 & 0,58 \\
\hline $\begin{array}{l}\text { Estado de desarrollo } \\
\text { de la enfermedad }\end{array}$ & $\begin{array}{l}\text { Severo } \\
\text { No severo }\end{array}$ & $\begin{array}{l}11 \\
84\end{array}$ & $\begin{array}{c}6 \\
79\end{array}$ & $1,72(0,56-5,54)$ & 1,07 & 0,30 \\
\hline Realización de AVD & $\begin{array}{l}\text { Dependiente } \\
\text { Independiente }\end{array}$ & $\begin{array}{l}32 \\
63\end{array}$ & $\begin{array}{l}19 \\
66\end{array}$ & $1,76(0,86-3,62)$ & 2,84 & 0,09 \\
\hline $\begin{array}{l}\text { Continuidad } \\
\text { de cuidado }\end{array}$ & $\begin{array}{l}\text { Permanente } \\
\text { No permanente }\end{array}$ & $\begin{array}{l}66 \\
29\end{array}$ & $\begin{array}{l}71 \\
14\end{array}$ & $0,45(0,20-0,97)$ & 4,87 & $0,02^{*}$ \\
\hline Cuidador Único & $\begin{array}{l}\text { Si } \\
\text { No }\end{array}$ & $\begin{array}{l}54 \\
41\end{array}$ & $\begin{array}{l}55 \\
30\end{array}$ & $0,72(0,38-1,37)$ & 1,16 & 0,28 \\
\hline
\end{tabular}

* Estadísticamente significativo a valor de $p=0,05$ 
un recurso vital para realizar cuidados domiciliarios con calidad, en tal sentido se requiere de experiencia y capacidad para asumir este rol y orientar el cuidado integral de la enfermedad, con el fin de evitar complicaciones (Burgos 2007, Figueroa 2010). De esta manera se destaca la importancia de los cuidados informales dados por las mujeres en el domicilio como un aporte significativo al bienestar de las personas con enfermedad crónica que contribuye también a la satisfacción de necesidades del núcleo familiar.

Teniendo en cuenta lo anterior, las etapas del ciclo vital en que se encuentran la mayoría de los cuidadores de adulto joven y maduro, son fundamentales para fortalecer el proceso enseñanza/aprendizaje con respecto a las acciones de cuidado, centradas en la promoción de la salud, prevención de la enfermedad y fortalecimiento del autocuidado, por lo que se recomienda orientar acciones con este propósito por medio de capacitación programada que atienda las particularidades de las características y de las necesidades de los cuidadores informales.

Otro aspecto que se destaca es el estado civil de los cuidadores, la mayoría son casado o viven en unión libre, aspecto que puede verse como una fuente de apoyo emocional y material importante, para tolerar el estrés, la ansiedad y la angustia que desencadenan los cuidados de larga duración y que ponen en riesgo la salud física y emocional del cuidador (Rodriguez et.al. 2005).

\section{Perfil educativo}

Al igual que en otros estudios, la escolaridad de los cuidadores es muy variada con tendencia a ser baja, sin embargo la edad y el nivel educativo del grupo de cuidadores participantes en el estudio, se considera un escenario funcional para fortalecer el conocimiento y la formación del cuidador en la búsqueda de un adecuado desempeño del rol, brindando un cuidado integral al sujeto de cuidado y demás miembros del grupo familiar (Burgos 2008). Así mismo el resultado de la preparación específica para brindar cuidado, es compatible con otros estudios (Ferrer 2008, Moreira de Souza et. al. 2011), que señalan que existe un alto porcentaje de cuidadores que no han recibido ningún tipo de capacitación y por lo tanto no están preparados para asumir esta responsabilidad del manejo de una situación crónica en el hogar.

En cualquier situación formal o informal, cuidar significa contar con los conocimientos, las habilidades y la experiencia para actuar con seguridad y planificar cuidados tendientes a promocionar la salud, prevenir complicaciones y fortalecer el autocuidado y la independencia de las personas a quienes se les brinda cuidado (Gonzalez et.al.2009). En este marco radica la importancia de fortalecer los conocimientos del cuidador informal, con el fin de favorecer la seguridad del paciente, fortalecer la habilidad de cuidado y la toma de decisiones responsables, para mantener a la persona en las mejores condiciones de salud y procurar una mejor calidad de vida (Sánchez et.al. 2008).

La presencia cada vez más creciente de enfermedades crónicas en los diferentes contextos y con ellas el aumento de la responsabilidad familiar frente al cuidado y seguimiento de la situación crónica, reafirman la responsabilidad social de los profesionales de enfermería para apoyar, preparar y asesorar el cuidado en el hogar (Torres et.al. 2008), con el fin de mejorar la habilidad de los cuidadores informales, quienes en su mayoría se caracterizan por tener conocimientos deficientes, manejo inadecuado de la enfermedad, altos niveles de estrés, de sobrecarga y déficit en su autocuidado, que en su conjunto conllevan a una percepción negativa de la enfermedad, aspecto que no contribuye a logar la adaptación positiva a la enfermedad, como plataforma para el desarrollo de un cuidado eficaz en el hogar.

Por tal razón, se requiere de la intervención educativa y del acompañamiento a los cuidadores informales con el fin de desarrollar sus potencialidades para fortalecer el rol de cuidador y mejorar la habilidad de cuidado de sí mismo, evitando la sobrecarga, el abandono personal, la generación de enfermedades y el deterioro de la calidad de vida.

\section{Perfil de salud}

En general los cuidadores consideran que gozan de buena salud, esta situación puede estar directamente relacionada con el nivel de independencia que aún tienen la mayoría de sujetos de cuidado. Un gran porcentaje cuenta con afiliación al Sistema General de Seguridad Social en Salud, aspecto que garantiza la atención y el cuidado en cualquier circunstancia de alteración de la salud. Es indiscutible que la sobrecarga del cuidador informal, afecta su salud física y mental, así como su desempeño en el rol, el cual se ve reflejado en el aumento del estrés, irritabilidad y hasta el abandono del sujeto de cuidado (Carretero et.al. 2006). Sin embargo, la situación encontrada en el estudio es diferente, los cuidadores en general gozan de buena salud, consideran que pueden trabajar y realizar las actividades de la vida diaria sin presentar mayores síntomas que alteren su salud y bienestar. 
El perfil de salud encontrado, se considera como algo positivo para el desempeño del rol, teniendo en cuenta que hasta ahora la mayoría de los cuidadores no perciben una alteración real de su salud, sin embargo se hace necesario fortalecer el autocuidado del cuidador informal, con el fin de empoderarlo hacia el manejo adecuado de su salud y prevenir los riesgos que sin duda traerán incremento de morbilidad y efectos negativos en su salud (Larrañaga et.al. 2008). La literatura establece que los programas de apoyo al fortalecimiento de la salud del cuidador, están dirigidos fundamentalmente a mejorar su calidad de vida, a través de la promoción de la actividad física, el descanso, la interacción social, el autoestima, el autocuidado y las redes de apoyo social, de tal forma que estos aspectos amortigüen los riesgos a que se ve enfrentado cotidianamente el cuidador frente a la situación de cronicidad (Pérez 2006, Ballester et.al. 2006).

Diversos autores (Carretero et.al. 2006 y Leal 2008), establecen que el componente educativo y el asesoramiento de los cuidadores, tienen efectos positivos sobre la salud y la calidad de vida de los cuidadores, a partir de la adopción de conductas que promueven el autocuidado, la autodeterminación y mejoran la habilidad para el cuidado de sí mismo y de otros. Por estas razones, se reconoce que el estado de salud de los cuidadores es un aspecto fundamental para el desempeño adecuado del rol, por lo que se recomienda el diseño y desarrollo de acciones preventivas con el fin de evitar la aparición de enfermedades que aumentarían sin ninguna duda, los indicadores regionales de cronicidad.

\section{Desempeño del rol}

En concordancia con otros estudios, la mayoría de los participantes son únicos cuidadores, con relación familiar con el sujeto de cuidado, aspecto que fortalece la responsabilidad, la creatividad y el afrontamiento para adoptar medidas frente al apoyo social, emocional y económico que requiera la familia, para avanzar de una manera activa y positiva en el cuidado de la salud de su ser querido (Barrera 2006).

De hecho, los resultados de la relación entre la condiciones de ser cuidador único y de mantener continuidad en el cuidado del enfermo muestran una asociación protectora y significativa en el caso de la presencia de cuidado permanente frente al nivel de calidad de vida del cuidador por lo que se considera que el entorno de cuidado continuo permitiría el desarrollo de respuestas adaptativas positivas por parte del cuidador frente al desempeño de su rol de manera que se cumplan con las expectativas de cuidado en una situación de control, de aprendizaje que favorece la toma de decisiones apropiadas para el enfermo, el cuidador y su entorno familiar.

Así, aún cuando está suficientemente referenciado en la literatura científica que cuidar enfermedades de larga duración, genera situaciones importantes de estrés y afectaciones de la salud física y mental, principalmente en el familiar que ha asumido la tarea de cuidar (Carretero et.al. 2006, Crespo 2007) algunos autores resaltan aspectos positivos como la satisfacción personal y familiar, la vivencia de sentimientos de placer y de gratificación, fortalecimiento del autoestima y de las relaciones interpersonales entre paciente y cuidador, mejoras en las relaciones familiares y mayor confianza en el desempeño del rol, producto de la dedicación, esfuerzo individual y familiar y ayuda prestada al familiar enfermo, que tiene como fin último el logro del bienestar, el mejoramiento en la calidad del cuidado y fortalecimiento del rol de cuidador (Carretero et.al. 2006).

Cuidar a un familiar significativo en la vida de las personas como padres, hijos, hermanos, abuelos y esposos, permite hacer visible el cuidado humanizado planteado por J. Watson, en el cual se evidencian diversos aspectos como el compromiso, la dedicación, el respeto, empatía, conocimiento profundo de las personas, reciprocidad e interacción que facilita un clima de confianza para compartir sentimientos, emociones y vivencias que favorece el crecimiento de los involucrados en la relación de cuidado (CebaIlos 2010). El conocimiento de la situación, el afecto, la confianza entre el sujeto de cuidado y cuidador y la responsabilidad que se experimenta frente al cuidado de la salud del familiar enfermo, se convierte en un escenario fundamental para brindar un cuidado oportuno y eficaz, para mantener la salud, prevenir complicaciones y mantener un trato digno e integral a la persona a quien se cuida. Lo anteriormente expuesto permite comprender lo expresado por los cuidadores sobre la facilidad que tiene para realizar la labor de cuidador, motivados por el deseo de proteger, cuidar y mantener la integridad de sus seres queridos, con el fin de hacer frente a las adversidades propias de la enfermedad crónica.

Por lo anterior, es fundamental reflexionar sobre los aspectos positivos del cuidado, que permiten el crecimiento personal, el desarrollo de potencialidades y fortalecimiento del autoestima, con el fin de que sean reconocidos y valorados por el propio cuidador para fortalecer el desempeño de su rol. 


\section{Agradecimientos}

Las autoras agradecen a la Universidad de los Llanos por el soporte financiero para el desarrollo de esta investigación suministrado a través de la Dirección General de Investigaciones de la Universidad de los Llanos. Agradecen también a los estudiantes del Programa de Enfermería por su participación en el desarrollo de este estudio como estudiantes en opción de grado.

\section{Referencias}

Aravena J, Sanhueza O. 2010. Evaluación de La Sobrecarga de Cuidadoras Informales. Revista Ciencia en Enfermería vol.16, n.3 [citado Oct. 9 de 2011]. URL: http://www.scielo.cl/scielo. php?pid=S0717-95532010000300012\&script=sci_arttext.

Ballester FD, Juvinyà C, Brugada M, Doltra J, Domingo R. 2006. Cambios en los cuidadores informales en cuanto a estilos de vida, relaciones y alteraciones de salud mental. Revista Presencia 2(4). [Consultado Octubre 11 de 2011] URL: http://www. index-f.com/presencia/n4/r56articulo.php.

Barrera L. et al. Habilidad de Cuidadores familiares de personas con enfermedad crónica. Mirada internacional. 2006. Revista Aquichan Vol 6 No. 1. [Consultado Octubre 11 de 2011]. URL: http://dikaion.unisabana.edu.co/index.php/aquichan/article/ viewArticle/77/159.

Bautista LM. 2008. Percepción de la calidad del cuidado de Enfermería en la ESE Francisco de Paula Santander. Revista Aquichan vol. 8 No. 1, [Consultado Octubre 9 de 2011]. URL: http://revistas.unisabana.edu.co/index.php/aquichan/article/ view/125/250.

Burgos P. et al. 2007. Nivel de conocimiento del cuidador informal de usuario con dependencia severa, adscrito al consultorio UItra estación de Chillán. Universidad del Bio Bio, Facultad de Ciencias de la salud, Escuela de Enfermería. Ministerio de Salud, Chile. [Consultado Agosto 18 de 2011]. URL: http://www. slideshare.net/polyburgos/o-b-j-e-t-i-v-o.

Burgos P. et al. Caracterización y nivel de conocimiento del cuidador informal de usuarios con dependencia severa adscritos al centro de salud Violeta Parra- Chillan. 2008. Universidad del Bio Bio, Facultad de Ciencias de la salud, Escuela de Enfermería. Ministerio de Salud, Chile. Revista Theoría, vol 17 No.2. [Consultado Octubre 10 de 2011]. URL: http://redalyc.uaemex.mx/pdf/299/29911533002.pdf.

Carretero S, Garcés FJ, Rodenas RF, San José LV. 2006. La sobrecarga de las cuidadoras de personas dependientes. Análisis y propuestas de intervención Psicosocial. 1 edición. Editorial Tirant Lo Blanch, Valencia España. p. 444

Ceballos VPA. 2010. Desde los ámbitos de Enfermería, analizando el cuidado humanizado. Revista Ciencia y Enfermería, vol 1 n.16. Concepción. [Consultado octubre 10 de 2011]. URL: http://www.scielo.cl/scielo.php?script=sci_arttext\&pid=S071795532010000100004\&lng=es\&nrm=iso\&tlng=es.
Crespo LM, López MJ. 2007. El estrés en cuidadores de mayores dependientes, Cuidarse para Cuidar. Ediciones. 1 edición. Editorial Pirámide., Madrid España. p. 278.

Espín A. 2008. Caracterización psicosocial de cuidadores informales de adultos mayores con demencia. Escuela Latinoamericana de Medicina. Cuba. [Consultado Agosto 6 de 2011]. URL: http:// bvs.sld.cu/revistas/spu/vol34_3_08/spu08308.htm.

Ferrer ME. 2008. El aprendizaje de cuidados familiares a pacientes con Alzheimer. Tesis doctoral Universidad de Alicante. Departamento de Enfermería, Alicante. [consultado Octubre 9 de 2010]. URL: http://rua.ua.es/dspace/bitstream/10045/9664/1/ tesis_elena_ferrer.pdf.

Gallardo G, Luna P, Marín M. 2004. Carencia de programas dirigidos a los cuidadores de adultos mayores. Diplomado en Salud Pública y Salud Familiar, Chile 2004. [Consultado Agosto 6 de 2011]. URL: http://medicina.uach.cl/saludpublica/diplomado/ contenido/trabajos/1/La\%20Serena\%202004/Carencia_de_ programas_dirigidos_a_los_cuidadores.pdf.

García M. et al. 2004. El sistema informal de cuidados en clave de desigualdad, informe SESPAS. Gaceta Sanitaria vol.18, suppl.1 [Consultado 2011-10-20], pp. 132-139. URL: http://scielo.isciii. es/scielo.php?pid=S0213-91112004000400021\&script=sci_ arttext.

González G, Rosa M, Bracho de L, Zambrano A, Marquina M, Guerra CA. 2009. El Cuidado Humano como valor en el ejercicio de los profesionales de la salud. [Consultado Octubre 9 de 2011]. URL: http://es.scribd.com/doc/16008571/Cuidado-Humano.

Larranaga I. et al. 2008. Impacto del cuidado informal en la salud y la calidad de vida de las personas cuidadoras: análisis de las desigualdades de género. Gaceta Sanitaria vol.22, n.5 [Consultado Octubre 11 de 2011]. URL: http://scielo.isciii.es/scielo. php?pid=S0213-91112008000500008\&script=sci_arttext.

Leal MI. Et al. 2008. Valoración de la sobrecarga en cuidadores informales de pacientes con Esquizofrenia, antes y después de un programa psicoeducativo. Actas Españolas de Psiquiatría 2008; 36 (2). [Consultado Octubre 11 de 2011]. URL: http://www. clinica.divisionescolpsic.org/articulos-docs/sobrecarga_familiares_esquizofrenicos.pdf.

Ministerio de Salud y Protección Social. Resolución 08430 de 1993. [Consultado Agosto 18 de 2012]. URL: http://www.dib.unal. edu.co/promocion/etica_res_8430_1993.pdf

Moreira de Souza R, Turrini RNT. 2011. Paciente Oncológico Terminal: Sobrecarga del Cuidador. Enfermería Global, revista electrónica trimestral de Enfermería. [Consultado octubre 9 de 2011]. URL: http://scielo.isciii.es/pdf/eg/v10n22/administracion2.pdf.

Ocampo JM, Herrera JA, Torres P, Rodriguez JA, Loboa L, García GA. 2007. La sobrecarga asociada con el cuidado de ancianos dependientes. Colombia Médica, Universidad del Valle. Vol 38, No. 1. [Consultado septiembre 22 de 2010].URL: http://colombiamedica.univalle.edu.co/Vol38No1/html/v38n1a6.html

Perez A. El cuidador primario de familiares con dependencia: Calidad de vida, apoyo social y salud mental, Facultad de medicina, Universidad de Salamanca. [en línea] 2006. [Consultado Octubre 11 de 2011]. Disponible en: http://www.imsersomayores. csic.es/documentos/documentos/perez-cuidador-01.pdf. 
Prieto A. 2007. Los cuidadores de pacientes con Alzheimer y su habilidad de cuidado en la ciudad de Cartagena. Revista Avances en Enfermería vol. 24 (2). [Consultado junio 15 de 2010]. URL: http://www.enfermeria.unal.edu.co/revista/articulos/xxv2_9. pdf

Qurantes MAJ. Et al. 2009. Estilo de vida, desarrollo científicotecnico y obesidad. Revista Cubana de Salud Pública versión on line, vol 35 No.3 La Habana [Consultado junio 15 de 2010]. URL: http://scielo.sld.cu/scielo.php?pid=S086434662009000300014\&script=sci_arttext.

Revista Colombiana de Cardiología. 2007. Guías Colombianas para el diagnóstico y tratamiento de la Hipertensión Arterial, vol.13 supl.1. [Consultado agosto 6 de 2011]. URL: http://www.scc. org.co/Portals/0/GUIAS_HIPERTENSION_ARTERIAL.pdf

Rodríguez M. et al. 2005. Calidad de vida en pacientes renales hemodializados. Revista Ciencia en Enfermería, vol.11, n.2 [Consultado 2011-10-20]. URL: http://www.scielo.cl/scielo. php?pid=S0717-95532005000200007\&script=sci_arttext.
Romero SL. et al. 2011. Eficacia de las intervenciones de enfermería mediante un programa para el cuidado en el hogar. Revista Cubana Enfermería, Vol 27 No.1 [Consultado octubre 12 de 2011]. URL: http://scielo.sld.cu/scielo.php?pid=S086403192011000100004\&script=sci_abstract.

Sánchez B. et al. 2008. Programa Cuidando a Cuidadores Familiares. Medwave, año VIII, No.11. [Consultado Oct.9 de 2011]. URL: http://www.mednet.cl/link.cgi/Medwave/Enfermeria/3663.

Torres E MP, Ballesteros PE, Sanchez CP. 2008. Programas de intervenciones de apoyo a los cuidadores informales en España. Revista Gerokomos, 19 (1) España. [Consultado agosto 17 de 2010]. URL: http://scielo.isciii.es/scielo.php?script=sci_ arttext\&pid=S1134-928X2008000100002.

Vaquiro S, Stiepovich J. 2010. Cuidado Informal, un reto asumido por la mujer. Revista Ciencia en Enfermería, vol.16, n.2 [Consultado septiembre 10 de 2011]. URL: http://www.scielo.cl/scielo.php?script=sci_arttext\&pid=S0717-95532010000200002. 